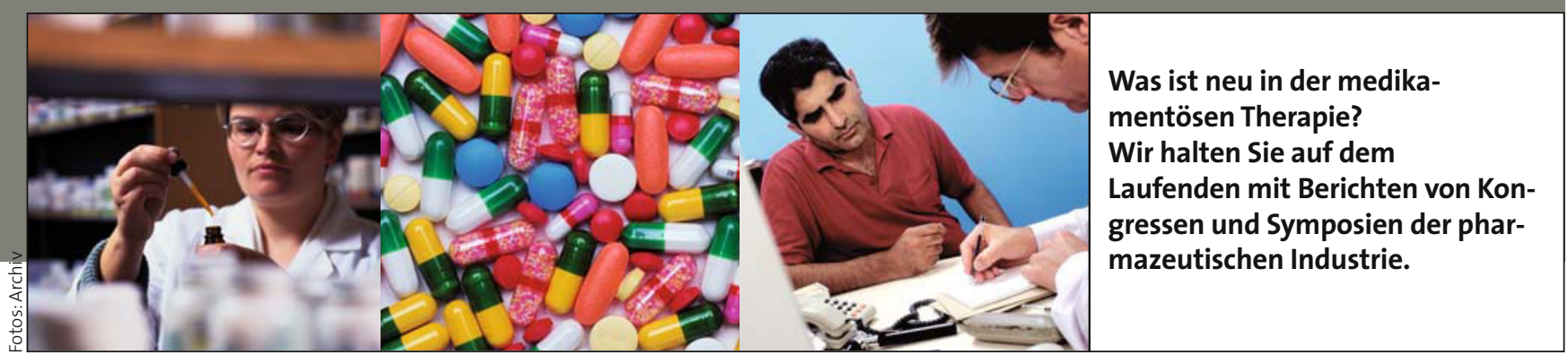

\title{
Alzheimerdemenz
}

\section{Was bringt die Frühdiagnose?}

- Der zur Alzheimerdemenz führende schleichende Krankheitsprozess wird zunehmend besser verstanden. Die begrenzte Voraussagekraft leichter kognitiver Störungen $(\mathrm{MCl})$ kann durch den Nachweis von Biomarkern im Liquor und bildgebende Verfahren erhöht und die Diagnose heute schon ,bei geringer Demenzausprägung mit hoher Sicherheit gestellt" werden, erklärte Prof. Dr. Wolfgang Maier, Bonn. Leider stehen den wachsenden diagnostischen Möglichkeiten keine durchgreifenden Präventionsstrategien gegenüber. „Was nutzen also die Erkennung von Risikostadien und die prädiktive Diagnostik? Es resultiert ein ethisches Dilemma", so Maiers Fazit.

Betroffene aber werden durch die „Frühdiagnose“ und Aufklärung über ihre Erkrankung stigmatisiert, verunsichert und deprimiert, was eine vorzeitige Verschlechterung ihres Zustands zur Folge haben kann. „Es gibt viele Gründe, einem Demenzverdacht nicht nachzugehen“, so Prof. Dr. Ingo Füsgen, Velbert-Neviges. „Demenzdiagnostik und die postdiagnostische Phase sind für den Demenzkranken nicht unbedingt förderlich." Kein Wunder also, dass Alzheimerdiagnosen in der Regel eher spät gestellt werden. Wichtig aber ist die Diagnose für die Begleitung von Patienten und Angehörigen, Therapie- und Zukunftsplanung.

\section{Hoffen auf neuroprotektive Wirkstoffe}

Ansätze für eine neuroprotektive Wirkung verschiedener Substanzen gibt es. So schützt beispielsweise der NMDAAntagonist Memantin vor Exzitotoxizität und den durch Beta-Amyloid induzierten Veränderungen, verringert die Tau-Phosphorylierung, hemmt das Absinken des Acetylcholinspiegels, verhindert mito- chondriale Funktionsstörungen und wirkt antientzündlich. „Es handelt sich um Ergebnisse aus Tierversuchen“, unterstrich Füsgen. „Aber sie bedeuten Hoffnung, vielleicht doch in absehbarer Zeit einen Einstieg in die Neuroprotektion zu finden."

Die Therapie der Alzheimerdemenz wird immer ein komplexes Maßnahmenbündel umfassen müssen, wozu laut Leitlinie Medikamente wie Memantin (z.B. Axura ${ }^{\circledR}$, indiziert bei moderater bis schwerer Alzheimerdemenz) und Acetylcholinesterase-Hemmer gehören. Noch ist sie allerdings in erster Linie auf Verlauf und Symptomatik ausgerichtet, nicht auf die Ursachen der Erkrankung.

\footnotetext{
- Ursula Einecke

Quelle: 32. Workshop des Zukunftsforums Demenz, Mainz, 22. Februar 2010 (Sponsor: Merz Pharmaceuticals $\mathrm{GmbH}$ )
}

\section{Bipolar affektive Störung}

\section{Ein Neuroleptikum für alle Fälle}

- Das atypische Neuroleptikum Quetiapin in seiner lang wirksamen Formulierung (Seroquel Prolong ${ }^{\circledR}$ ) besitzt inzwischen auch die Zulassung zur Rezidivprophylaxe bei bipolaren Störungen. Damit kann es in allen Phasen der Erkrankung eingesetzt werden, von der in Deutschland etwa zwei Millionen Menschen betroffen sind.

Bipolare Störungen zählen nach Ansicht von Prof. Dr. Stephanie Krüger, Berlin, zu den maßlos unterschätzten Erkrankungen. Viel zu selten adäquat diagnostiziert werden insbesondere Bipolar-II-Störungen, bei denen sich depressive Phasen mit weniger stark ausgeprägten manischen Episoden - sog. Hypomanien - abwechseln. Verkannt werden diese Erkrankungen vor allem deshalb, weil sich Patienten in den subjektiv als durchaus angenehm empfundenen hypomanischen Phasen kaum an einen Arzt wenden. Tat- sächlich vermutet Krüger bei 30-50\% der Patienten mit der vermeintlichen Diagnose einer „rezidivierenden depressiven Störung" eine Bipolar-II-Störung.

\section{Folgenreiche Fehldiagnose}

Entsprechende Fehldiagnosen bleiben nicht folgenlos - zumal dann, wenn Patienten mit bipolaren Störungen eine Monotherapie mit Antidepressiva erhalten. Die Patienten haben in diesem Fall ein ho- 